\title{
A!
}

This is an electronic reprint of the original article.

This reprint may differ from the original in pagination and typographic detail.

Myllynen, Antti; Sadi, Toufik; Oksanen, Jani

\section{Current Spreading in Back-Contacted GalnP/GaAs Light-Emitting Diodes}

\author{
Published in: \\ IEEE Transactions on Electron Devices
}

DOI:

10.1109/TED.2020.2964662

Published: 01/03/2020

Document Version

Peer reviewed version

Please cite the original version:

Myllynen, A., Sadi, T., \& Oksanen, J. (2020). Current Spreading in Back-Contacted GalnP/GaAs Light-Emitting Diodes. IEEE Transactions on Electron Devices, 67(3), 1027-1033. [8972369].

https://doi.org/10.1109/TED.2020.2964662

This material is protected by copyright and other intellectual property rights, and duplication or sale of all or part of any of the repository collections is not permitted, except that material may be duplicated by you for your research use or educational purposes in electronic or print form. You must obtain permission for any other use. Electronic or print copies may not be offered, whether for sale or otherwise to anyone who is not an authorised user. 


\title{
Current Spreading in Back-Contacted GaInP/GaAs Light-Emitting Diodes
}

\author{
Antti Myllynen, Toufik Sadi, and Jani Oksanen
}

\begin{abstract}
The recently proposed diffusion-driven charge transport (DDCT) method can allow a paradigm shift in the design of optoelectronic devices, by changing both the current injection principle and the device structure. The DDCT injection technique is based on the bipolar electron and hole diffusion currents that are used to electrically inject charge carriers into an active region (AR) located outside the p-n junction. In this article, we study an interdigitated back-contacted DDCT-light-emitting diode (LED) based on a GaInP/GaAs double heterojunction (DHJ) structure consisting of lateral heterojunctions (LHJs) located above a uniform AR. The structure uses single-sided electrical injection and is suitable for large-area applications and thin-film devices with near-surface ARs. Our analysis, based on charge transport simulations, suggests that the structure permits more efficient current spreading and lower surface recombination than conventional structures, leading to a very high internal quantum efficiency (IQE) and injection efficiency exceeding $99 \%$. Particularly, we investigate the implications of using the new structure for improving the efficiency of LEDs, bringing them closer to the threshold of electroluminescent cooling (ELC). The results predict an above-unity internal power conversion efficiency for the DDCT-LEDs, substantially exceeding the efficiency of conventional reference devices, highlighting the new possibilities that DDCT devices offer especially for high-power ELC at room temperature.
\end{abstract}

Index Terms-Current spreading, diffusion-driven charge transport (DDCT), lateral heterojunction (LHJ), light-emitting diodes (LEDs).

\section{INTRODUCTION}

$\mathbf{T}$ HE performance of light-emitting diodes (LEDs) has increased significantly over the past few decades [1]. In terms of the external quantum efficiency (EQE), the best devices have already reached efficiencies of $81 \%$ for III-N materials [2], and 68\% for III-As materials [3]. Despite the enormous progress of the technology, there is still room for improvements especially for high-power lighting applications and emerging new possibilities such as thermophotonic or electroluminescent cooling (ELC) [4]. Achieving ELC is a timely challenge requiring extremely high LED EQEs. Although internal quantum efficiencies (IQEs) nearing 100\% have been reported for certain III-As devices [5], LED EQEs are currently limited by several factors, including efficiency droop, resistive losses, current crowding, and poor light extraction [1].

This work was supported by the Academy of Finland and the European Research Council under the Horizon 2020 research and innovation programme (grant agreement No 638173).

The authors are with the Engineered Nanosystems Group, School of Science, Aalto University, 00076 AALTO, Finland, (e-mail: antti.i.myllynen@aalto.fi; toufik.sade@aalto.fi).
In a traditional double heterojunction (DHJ) structure, the active region (AR) of the device is sandwiched between the pand $\mathrm{n}$-doped cladding layers. In this design, the electrons and holes are injected into the AR from opposite sides as illustrated with the solid lines in Fig. 1(a), showing a specific example of a flip-chip (FC) LED that constitutes the emitter part of a thermophotonic device prototype [6], [7]. The efficiencies of this specific design and other conventional FC-LEDs [2], [8], [9] are typically limited by the current spreading resistance in the n-type CSL, and the potentially strong surface recombination channels at the mesa edges, as illustrated with the dashed lines in Fig. 1(a). These channels are present in practically all ARs embedded within a p-n junction, either at the device edges and/or the contact vias through the $\mathrm{AR}$, as required to fabricate the n-contacts. This also applies to the thin-film (TF) LEDs [3], [10] where the lateral current spreading is improved by fabricating contacts on both sides of the device. This limits surface recombination to the device edges, but also leads to shading by the light-blocking top contacts.

The recently proposed diffusion-driven charge transport (DDCT) method [11]-[14] was suggested to reduce the current spreading limitations of conventional GaN-based FC-LED structures. The DDCT injection utilizes bipolar electron and hole diffusion currents to inject charge carriers into ARs located outside the p-n junction. This is possible via lateral heterojunctions (LHJs) [15]-[18] located above a uniform AR as illustrated in Figs. 1(b) and 1(c) for the GaInP/GaAs DDCT-LED studied in this work. The main current paths are illustrated in Fig. 1(c) with solid lines. Some surface recombination is expected also for the DDCT-LED, as illustrated with the dashed lines in Fig. 1(b) and 1(c). However, the surface channels of the DDCT-LED are almost negligible compared to the FC-LED of Fig. 1(a), due to the lateral doping and the modified structure as indicated by the results of this work. Generally, the DDCT-LEDs can enable novel devices if the substrate is removed with e.g., an epitaxial lift-off (ELO) process [19], [20]. For example, fabrication of back-contacted TF LEDs and near surface active regions should be possible. Also, with the ELO, one surface of the device would be completely free for further processing such as surface texturing [21], anti-reflection, or surface plasmon coatings [22], [23] that could be used to improve the light extraction from the DDCTLEDs.

One possible application where the benefits offered by the DDCT-LEDs can be particularly attractive is ELC [7], [10], [24]-[27], where the large non-radiative lifetimes of III-As materials [28] are a clear advantage over III-N materials. In ELC, the lattice heat absorption accompanying the sponta- 

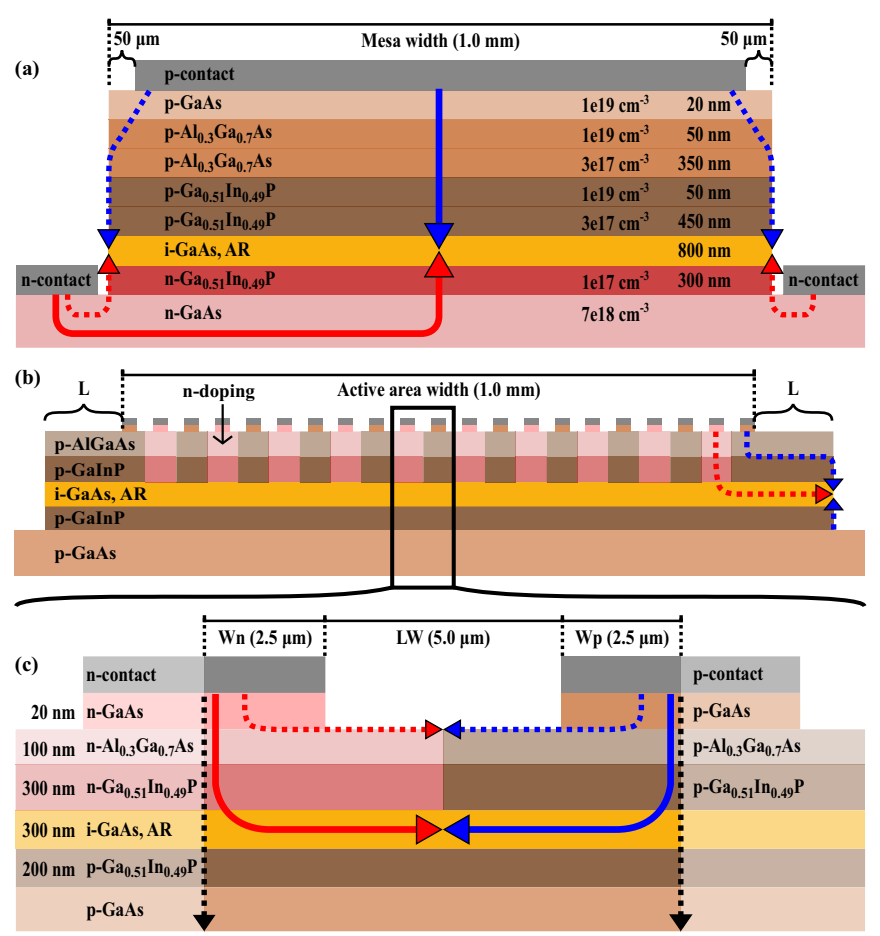

Fig. 1. (a) Schematic example of a conventional FC-LED. Electron (red) and hole (blue) current paths are illustrated for the main current (solid line) and the surface current (dashed line). (b) Interdigitated back-contacted DDCT-LED (c) Single period of the device with a LW of $5 \mu \mathrm{m}$. Doping concentration of $3 \times 10^{17} \mathrm{~cm}^{-3}$ and $1 \times 10^{18} \mathrm{~cm}^{-3}$ were used for $\mathrm{n}$ - and $\mathrm{p}$-doped regions, respectively. Also, the main and surface current paths are illustrated with solid and dashed lines, respectively. In addition, cut-lines (black dashed arrows) to be used in Fig. 4 for plotting the band diagrams are shown.

neous emission of light can lead to a new solid-state cooling effect in highly efficient devices [29]-[31], if the voltage and surface recombination losses can be eliminated [7]. Up until now, ELC has been demonstrated at very low powers [26], [27] and indirect evidence of possible internal cooling at high powers has recently been reported [6].

In this paper, we study the emerging possibilities of a very recently proposed laterally-doped GaInP/GaAs DHJ LED [32], using the DDCT concept and modulation doping (MD) [33], [34]. We use charge transport simulations to establish the practical requirements and limits of the new structure, and explore the effects of the lateral dimensions on device operation, especially lateral current spreading. Our results suggest that the GaInP/GaAs DDCT-LEDs might facilitate developing highpower ELC applications, such as thermophotonic heat pumps (THPs) [4] and double diode structures (DDSs) [7], [24]. In particular, we show how DDCT injection can be adapted for efficient high-power III-As LEDs that can be integrated in the DDS devices.

\section{Device Structure and Simulations}

Fig. 1 schematically illustrates the structures simulated in this work. The structure of Fig. 1(a) represents a reference FC-LED where surface recombination only takes place at the mesa edges. Fig. 1(b) equivalently shows the structure of a laterally-doped back-contacted DDCT-LED consisting of several periods of lateral diodes on top of a uniform AR adding up to a $1 \mathrm{~mm}$ effective device width. The distance $L$ between the edge of the mesa and the last period of the lateral diodes can additionally be freely adjusted. Fig. 1(c) shows the periodic DDCT unit in more detail and illustrates different current paths present in the device. The base structure of the device is a p-GaInP/i-GaAs/n-GaInP DHJ $(200 / 300 / 300 \mathrm{~nm})$ grown on a p-GaAs substrate, followed by a $100 \mathrm{~nm}$ thick $\mathrm{n}$-AlGaAs layer and a $20 \mathrm{~nm}$ thick $\mathrm{n}-\mathrm{GaAs}$ contact layer. The LHJs can be fabricated with a selective-area doping method, such as diffusion doping [35], [36] or ion-implantation [37], to form $\mathrm{p}$-doped regions in the n-doped layers above the AR. The p-regions extend ideally from the p-contact metal to the edge of the AR in the vertical direction. Also, the GaAs contact layer between the $\mathrm{n}$ - and p-contacts is considered to be removed, e.g., with a selective wet etching process, to minimize surface recombination at the free GaAs surface, and to eliminate the parasitic small bandgap diode that would otherwise be formed within the contact layer. The lateral dimension of the $\mathrm{n}$ - and $\mathrm{p}$-contacts, and the un-contacted area between the contacts are varied between $1 \mu \mathrm{m}$ and $20 \mu \mathrm{m}$ to provide insight on the trade-offs between the fabrication linewidth (LW) and device performance, within the limits of optical lithography.

When the DDCT-LED is forward-biased, the charge carriers are electrically injected into the lateral p-n junctions. The applied bias voltage of the LED will nevertheless also excite the GaAs AR, having a bandgap energy $\left(E_{g}\right)$ of $1.42 \mathrm{eV}$ at room temperature. Since the bandgap of GaAs is significantly lower compared to $\mathrm{Al}_{0.3} \mathrm{Ga}_{0.7} \mathrm{As}(1.88 \mathrm{eV})$ and $\mathrm{Ga}_{0.51} \mathrm{In}_{0.49} \mathrm{P}$ $(1.8 \mathrm{eV})$, the electrons and holes will preferentially diffuse into the AR where they spread, via drifting and/or diffusion, and recombine with each other. Since the maximum value of the applied bias $(\sim 1.42 \mathrm{~V})$ is justifiably limited to around the bandgap voltage of GaAs, the current leakage through the lateral $\mathrm{p}-\mathrm{n}$ junctions in the horizontal direction is negligible as confirmed by our simulations.

The structure of the reference FC-LED [see Fig. 1(a)] is a circular $1 \mathrm{~mm}$ diameter mesa consisting of a GaInP/GaAs DHJ structure, as part of a DDS device we are studying to demonstrate ELC [6], [7]. This structure has typically provided the highest efficiency for the device due to the minimized surface recombination enabled by the reduced surface-to-volume ratio. In the DDS context, the FC-LED is optically coupled to a GaAs homojunction photodiode (not shown here) enclosed within a single semiconductor epistructure. The aim of the DDS is to facilitate direct observation of high-power ELC by eliminating the need for light extraction. However, the device nevertheless suffers from the general challenges of current spreading and surface recombination seen in conventional LEDs. In optical terms, however, the DDS does not require light extraction from the semiconductor in ELC studies, in contrast to conventional LEDs. Also, the internal optical losses can be quite low, as a very high top surface reflectivity of the order of $99 \%$ can be achieved, e.g., by depositing a $\mathrm{Si}_{3} \mathrm{~N}_{4}$ film on the uncontacted areas and forming a $\mathrm{Si}_{3} \mathrm{~N}_{4} /$ metal omnidirectional reflector (ODR) structure at the contacts [24].

To simulate our structures at room temperature, we use a numerical transport model that has been extensively calibrated 
against experimental data to accurately describe the behavior of III-As LEDs and DDSs over wide operation regimes (see Sadi et al. [7], [38], [39]). The model is based on the well established drift-diffusion description [40]-[42], coupling the partial differential equations for the electrostatic potential and the quasi-Fermi levels for electrons and holes in the 2-D real space. The recombination rates are calculated using the wellknown ABC model for Shockley-Read-Hall (SRH), radiative, and Auger recombination [40] which provides a widely used and accurate description of the carrier recombination for nondegenerate carrier densities. We use well established literature values of the material parameters, including the constants $B \sim 2 \times 10^{-10} \mathrm{~cm}^{3} \mathrm{~s}^{-1}$ [43], [44] and $C \sim 1 \times 10^{-30} \mathrm{~cm}^{6} \mathrm{~s}^{-1}$ [45] for the radiative and Auger recombination, respectively. For the SRH recombination, we use a conservative recombination constant $A \sim 3 \times 10^{5} \mathrm{~s}^{-1}$, as compared to the best values reported for GaAs [28], as it represents the upper limit for the experimental model calibration sensitivity with our DDS device simulations [7]. The non-radiative surface and interface recombination rates at the free AlGaAs surface and $\mathrm{GaInP} / \mathrm{GaAs}$ interfaces, are calculated as discussed by Kivisaari et al. [41], using surface and interface recombination velocities of $\sim 4 \times 10^{5}$ and $1.5 \mathrm{~cm} / \mathrm{s}$ [46], respectively. Overall, our parameters correspond to a relatively high quality device, probing the potential performance and optimal design of the DDCT-LEDs, from the current transport point of view.

In analyzing our results, the IQE is defined as the ratio of the radiative recombination rate to the rate corresponding to the injection current. Therefore, the definition includes the current injection efficiency and corresponds to the overall quantum efficiency of generating photons in the device. Also, in analyzing the lateral current spreading, we define the uniformity of the total recombination rate in the AR of the device as the minimum rate of the recombination, averaged over the vertical direction, divided by the similarly averaged maximum rate. We have also defined the internal power conversion efficiency (IPCE) to correspond to the PCE in the absence of optical losses. This figure of merit is used to evaluate the electrical performance of the LEDs, and to estimate the potential of the DDCT-LEDs for ELC in the DDS context, where light extraction is essentially not needed. The IPCE is calculated as $\mathrm{IQE} \times\left(E_{g}+k_{B} T\right) / q V$ [38], where $E_{g}$ is the bandgap (and $E_{g}+k_{B} T \sim$ average photon energy), $k_{B}$ is the Boltzmann constant, $T$ is the temperature, $V$ is the LED bias, and $q$ is the elementary charge.

\section{RESUlTS AND DISCUSSION}

Fig. 2(a) shows the total current density $J$ and the IQE for the DDCT-LEDs for different LWs and the reference FCLED as a function of bias. In the $J-V$ curve of the DDCTLEDs, typical LED characteristics are observed, with an SRHlike recombination dominated region at low biases, and a radiative recombination dominated region at a bias range of 1-1.2 V. At higher voltages and current densities, the current becomes resistively limited. Generally, the current density decreases slightly as the LW increases, due to less efficient carrier diffusion. The $J-V$ characteristics of the reference FCLED are rather different due to the approximately 3 orders of
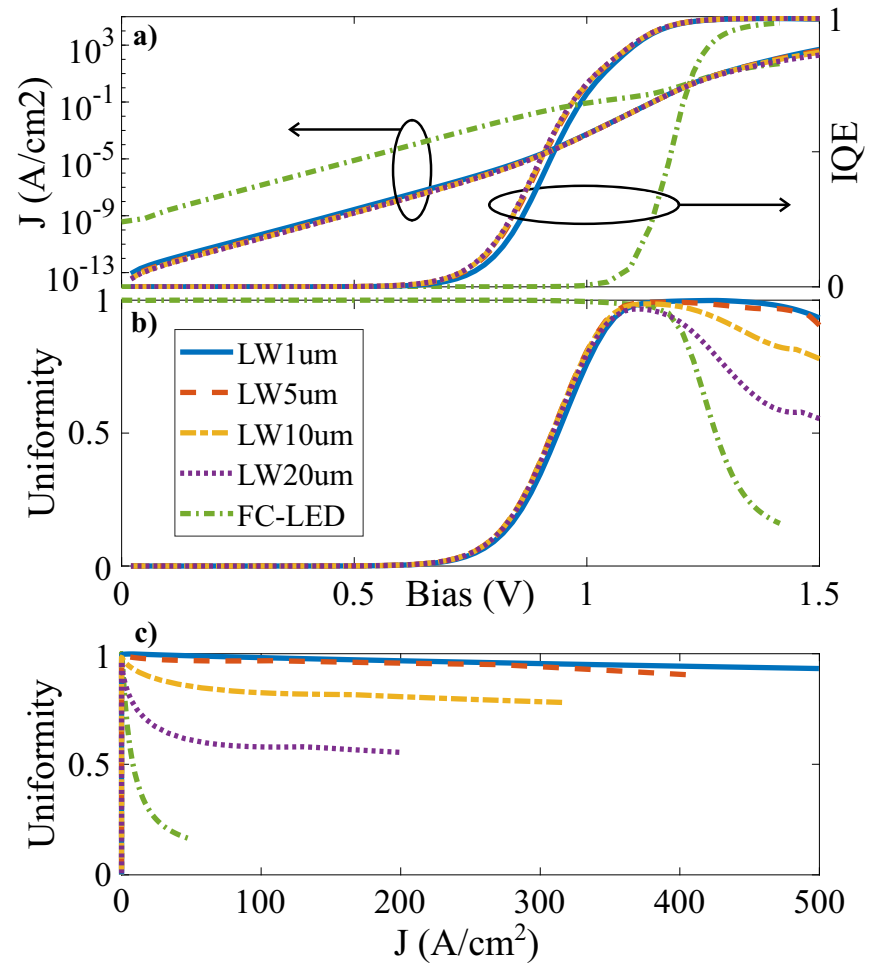

Fig. 2. Results for the DDCT-LEDs, with a LW of 1, 5, 10, and $20 \mu \mathrm{m}$, and the reference FC-LED, showing (a) the $J-V$ curves (left axis) and the IQE (right axis) as a function of bias. Also, the recombination uniformity of the devices is shown as a function of (b) bias and (c) current density.

magnitude higher current density at low biases. This is the result of a very strong surface recombination that occurs at the DDS mesa edges, as illustrated in Fig. 1(a). At $\sim 1 \mathrm{~V}$, the surface recombination current starts to saturate and the current density starts to approach that of the DDCT-LEDs.

Fig. 2(a) shows also that the IQE of all structures is practically zero at low voltages due to the dominant SRH recombination. Above $\sim 0.6 \mathrm{~V}$, the IQE of the DDCT-LEDs starts to increase with the radiative recombination rate. The IQE of the DDCT devices exceeds $99 \%$ at a large bias range of 1.28-1.5 V peaking at $99.3 \%-99.4 \%$ depending on the LW. After the peak, the IQE only decreases very slightly. It is noteworthy that the higher recombination rate observed for the smallest LW of $1 \mu \mathrm{m}$ leads to slightly lower IQE at the bias range of $\sim 0.6-1.1 \mathrm{~V}$. The IQE of the reference FCLED exceeds $97 \%$ at biases larger than $1.38 \mathrm{~V}$, peaking at 97.7\%. The main differences between the structures originate from the surface and SRH recombination rates. In the DDCTLEDs, most of the non-radiative surface recombination at the mesa edges can be efficiently suppressed by increasing the separation $L$ between the edge and the outermost p-contact. Further analysis indicates that an edge separation $L$ of $200 \mu \mathrm{m}$ efficently blocks the electron diffusion to the surface, and is sufficient for eliminating practically all surface recombination in the DDCT structure. This is a clear advantage of the new structure over the reference FC-LED, where similar edge separation is not useful because the AR is placed between the $\mathrm{p}$ - and n-doped layers that provide a conductive channel for both charge carriers to the edge, even if the separation 
distance is large. In addition to surface recombination, further differences in the performance of the structures are attributed to the SRH recombination, which is reduced in the DDCT structure at lower biases $(0.6 \mathrm{~V}-1.1 \mathrm{~V})$ due to more favourable carrier statistics. In this structure, most of the $\mathrm{SRH}$ recombination occurs in the depletion region that forms between the effectively $n$ - and p-doped regions in the AR. Therefore, as opposed to the reference FC-LED where the depletion region extends throughout the AR, the volume where SRH recombination occurs is smaller for the DDCT-LED and consequently the SRH recombination extent is lower.

Fig. 2(b) shows the recombination uniformity of the LEDs as a function of bias. The uniformity of the DDCT-LEDs is essentially zero at low biases, due to inefficient electron spreading in the electron depleted AR. Further analysis indicates that the SRH recombination at low biases is occurring mainly below the n-doped region, since electrons will recombine before they are able to spread in the AR. The uniformity starts to increase with the IQE when the electron density in the AR increases, leading to very uniform radiative recombination over a relatively large range of biases. For the DDCT-LED with a LW of $5 \mu \mathrm{m}$ a maximum uniformity of $99.3 \%$ was achieved at $1.16 \mathrm{~V}$. For the other structures, the maximum uniformity is observed at $\sim 1.1-1.35 \mathrm{~V}$, depending on the LW. The decreasing uniformity after the peak shows rather distinct characteristic for different LWs. With small linewidths of $\leq 5 \mu \mathrm{m}$ we observe a small amount of conventional current crowding at large currents where the recombination is focused under the p-doped region due to the low hole mobility limiting the current spreading. In contrast to this, the larger linewidths in excess of $10-\mu \mathrm{m}$ lead to unconventional current crowding where the recombination is strongly pronounced under the ndoped region. This is caused by the diffusion of electrons remaining less efficient than the transport of holes that is aided by the MD. The uniformity of the reference FC-LED structure is essentially $100 \%$ at voltages below $0.95 \mathrm{~V}$ due to the uniform electron and hole injection enabled by the constant doping on the opposite sides of the AR. In contrast to the DDCT-LEDs, the uniformity of the reference structure starts to decrease already when the IQE begins to increase as the very large current density in the n-CSL leads to a very strong current crowding at the p-contact edge. It is nevertheless evident that the current crowding effects in the FC-LED could be significantly reduced by the TF-LED configuration. However, even in this case, the surface recombination effects would remain essentially identical, and the contact grid on the other side of the AR would shade a substantial portion of the surface and prevent the structures use, e.g., in the selected thermophotonic applications.

Fig. 2(c) represents the recombination uniformity of the devices as a function of the current density. The uniformity of the DDCT-LEDs with a LW of $\leq 5 \mu \mathrm{m}$ decreases slowly and almost linearly as $J$ increases. With larger LWs of $\geq 10 \mu \mathrm{m}$, the curve exhibits different features where the uniformity decreases first almost exponentially before reaching a more linear region. The uniformity of the FC-LED decreases also exponentially but even more rapidly if compared to the lowest performing DDCT-LED with a LW of $20 \mu \mathrm{m}$. Additionally,

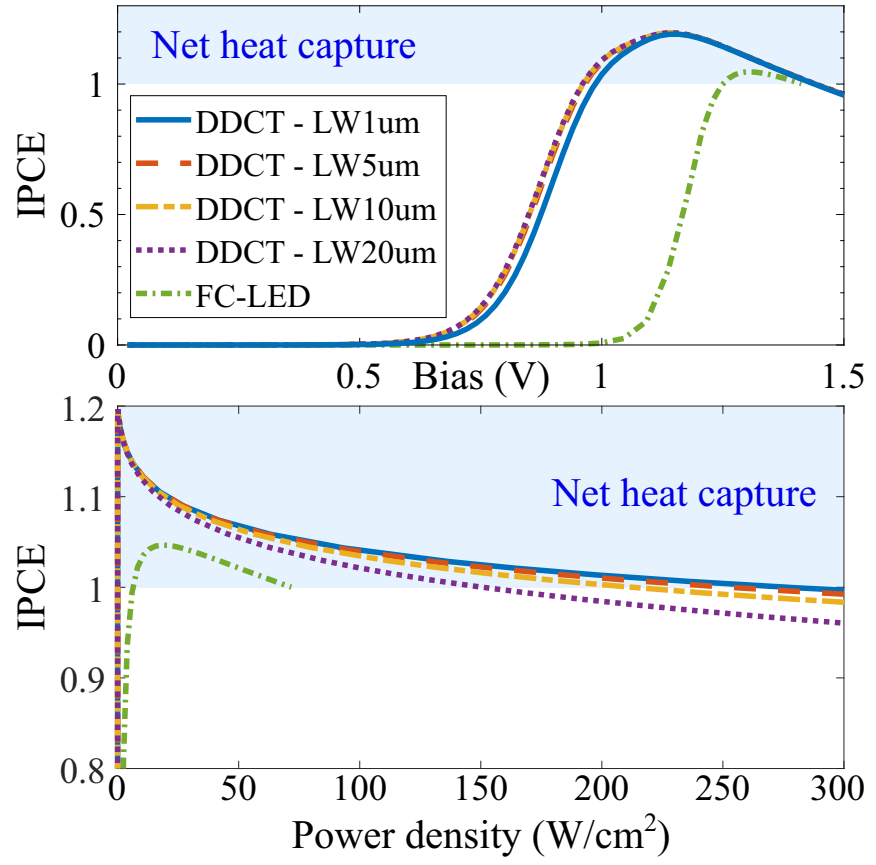

Fig. 3. IPCE of the DDCT-LEDs and the reference FC-LED as a function of bias and power density, indicating that ELC should be substantially easier to observe with the new DDCT designs.

Fig. 2(c) shows the maximum current density of the DDCTLEDs, reached at the bias of $1.5 \mathrm{~V}$. The maximum current significantly reduces with increasing LW due to increased current spreading resistance in the AR. Overall, these results suggest that an optimal combination of high uniformity and IQE is achieved with a $\mathrm{LW}$ of $5 \mu \mathrm{m}$. For the reference FCLED, the maximum bias voltage was limited to the bandgap energy of GaAs, as higher biases are not interesting in the DDS context and the uniformity reached very low values already at this voltage.

Fig. 3 shows a comparison of the IPCE of the devices as a function of bias voltage and power density. For the DDCT-LEDs the cooling regime where IPCE exceeds $100 \%$ is reached for a large power range from $0.12 \mathrm{~mW} / \mathrm{cm}^{2}$ to $150-270 \mathrm{~W} / \mathrm{cm}^{2}$, depending on the LW of the structure. The peak values of $\sim 120 \%$ are achieved at a power density of $\sim 0.1 \mathrm{~W} / \mathrm{cm}^{2}$ for all DDCT devices. For the reference FCLED, cooling regime is observed on a significantly narrower power range, and also the peak value of $\sim 105 \%$ is noticeably smaller. This difference originates from the strong surface recombination that reduces the IQE of the FC-LED. These results indicate that both designs could achieve ELC from the electrical point of view. However, with the DDCT-LEDs, the cooling should be substantially easier to achieve and become possible already at a light extraction efficiency of $\sim 80 \%$, as compared to the minimum of $\sim 95 \%$ required with conventional LED designs.

Fig. 4 analyzes the carrier densities of the DDCT-LEDs under the $\mathrm{n}$ - and p-contacts in more detail, showing their values in the vertical direction along the dashed black arrows illustrated in Fig. 1(c). The results are presented for LWs of 5 and $20 \mu \mathrm{m}$ at the operation voltages of $1.16 \mathrm{~V}$ and $1.12 \mathrm{~V}$, 


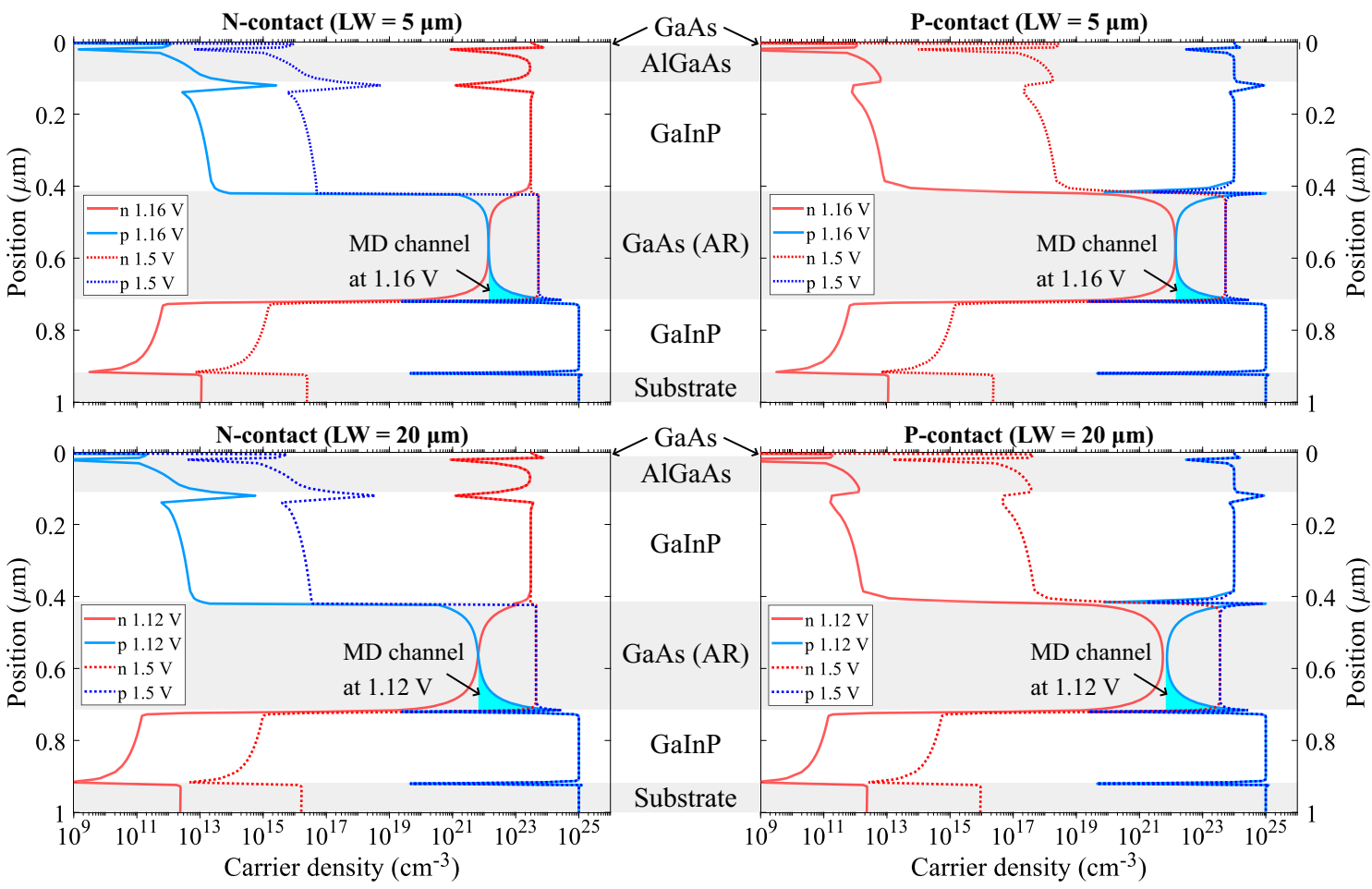

Fig. 4. Carrier distribution in vertical direction at the middle of $n$ - and p-contacts, following the cut-lines illustrated in Fig. 1(b), for structures with LW of 5 $\mu \mathrm{m}$ and $20 \mu \mathrm{m}$. A modulation doping (MD) channel is observed at the lower GaAs/GaInP interface at maximum of the uniformity reached at 1.16 and 1.12 $\mathrm{V}$ for LW of $5 \mu \mathrm{m}$ and $20 \mu \mathrm{m}$, respectively. At maximum bias of $1.5 \mathrm{~V}$ the MD channel is significantly smaller, due to higher and almost uniform carrier density within the AR. This indicates that the effect of MD is decreasing at high biases.

respectively, corresponding to the biases required for the maximum uniformity, and at the maximum bias of $1.5 \mathrm{~V}$. The subfigures on the left panel of Fig. 4 show the carrier densities under the $\mathrm{n}$-contact, where the layer structure resembles a $\mathrm{p}$ $\mathrm{n}$ junction due to the MD. The minority carrier densities in the $\mathrm{p}$ - and $\mathrm{n}$-doped regions remain many orders of magnitude smaller than the majority carrier densities, indicating that recombination is negligible outside the AR. Below the pcontact (on the right panel) electrons are the minority carriers on both sides of the AR, and their density also remains small. The results show that the MD is present in a narrow channel next to the lower GaAs/GaInP interface. Particularly, this MD channel is observed in both structures and under both contacts at the operation voltage of maximum uniformity. At a bias of $1.5 \mathrm{~V}$, the MD channel is much less pronounced due to the significantly higher carrier concentrations within the AR. Further analysis indicates that, in the $20-\mu \mathrm{m} \mathrm{LW}$ structure, the carrier densities in the AR under p-contact are slightly smaller as compared to n-contact. This reflects the reduced recombination uniformity, even though this is not clearly visible in Fig. 4 due to the very large carrier density range.

In addition to the completely single-sided contacts and fully scalable structure, the main advantage of the DDCT-LEDs, as compared to the reference FC-LED, is the strong minimization of surface recombination at the mesa edge. This advantage is present without any surface passivation and it is made possible by the lateral current injection and the unconventional device structure where all the barrier layers close to the edge are p- doped. In this structure, the n-doped conductive channel to the edge is completely absent and electrons have a reduced access to the edge. As discussed by Sadi et al. [7], surface passivation could be applied to the reference FC-LED to improve its performance. However, even with perfect surface passivation the performance of the FC-LEDs will not reach the performance of DDCT-LEDs, due to the additionally reduced SRH recombination of the DDCT structure. In general, the results presented here showed that the recombination uniformity decreases as the LW of the devices is increased. Similar findings were reported for GaN DDCT devices by Kivisaari et al. [15]. However, for the GaN devices a reduction in the recombination rate under the n-doped region was observed for all practical device dimensions. This reduction is not pronounced in our DDCT devices if the LW is $10 \mu \mathrm{m}$ or less, highlighting their exceptional recombination uniformity enabled by the MD from the p-GaInP layer.

The proposed DDCT structure is promising for multiple applications, such as high-efficiency LEDs and ELC, and it provides also interesting possibilities for back-contacted solar cells. Additionally, large-area devices should be easy to realize by repeating a small DDCT-unit to form large continuous arrays while maintaining excellent device performance. However, there is still room for further optimizations. For example, the small amount of unconventional current crowding that was observed under the $\mathrm{n}$-contact in devices with larger contact dimensions indicates that the MD level should be individually optimized for each structure, within the limits of the epitaxial growth process and the achievable doping 
levels. Also, the results suggest that the uniformity of the larger devices could be further improved by reducing the doping level of the p-GaInP MD layer. Overall, the analysis indicates that the laterally-doped DDCT-LEDs with single-sided electrical injection may provide clear improvements compared to the conventional FC-LEDs [6], [7], opening new possibilities for high-efficiency large-area lighting and high-power solid-state cooling.

\section{CONCLUSIONS}

In conclusion, we have shown that a back-contacted laterally-doped $\mathrm{GaInP} / \mathrm{GaAs}$ DDCT-LED with p-type MD could simultaneously eliminate current crowding, and provide an excellent IQE exceeding 99\%, as enabled by minimized surface recombination. The results also suggested that the DDCT-LEDs had a great potential to improve the performance of the DDSs that were used to study ELC, by providing aboveunity IPCE for a large power density range of $0.12 \mathrm{~mW} / \mathrm{cm}^{2}-$ $270 \mathrm{~W} / \mathrm{cm}^{2}$. Furthermore, our device design was scalable for large-area applications.

\section{REFERENCES}

[1] S. Nakamura and M. R. Krames, "History of Gallium-NitrideBased Light-Emitting Diodes for Illumination," Proceedings of the IEEE, vol. 101, no. 10, pp. 2211-2220, Oct 2013, doi: 10.1109/JPROC.2013.2274929.

[2] C. A. Hurni, A. David, M. J. Cich, R. I. Aldaz, B. Ellis, K. Huang, A. Tyagi, R. A. DeLille, M. D. Craven, and F. M. Steranka, "Bulk GaN flip-chip violet light-emitting diodes with optimized efficiency for high-power operation," Appl. Phys. Lett., vol. 106, p. 031101, 2015, doi: $10.1063 / 1.4905873$.

[3] M. Broell, P. Sundgren, A. Rudolph, W. Schmid, A. Vogl, and M. Behringer, "New developments on high-efficiency infrared and InGaAlP light-emitting diodes at OSRAM Opto Semiconductors," Proc. SPIE, vol. 9003, p. 90030L, 2014, doi: 10.1117/12.2039078.

[4] J. Oksanen and J. Tulkki, "Thermophotonic heat pump - a theoretical model and numerical simulations," J. Appl. Phys., vol. 107, no. 9, p. 093106, May 2010, doi: 10.1063/1.3419716.

[5] D. Ban, H. Luo, H. C. Liu, Z. R. Wasilewski, A. J. SpringThorpe, R. Glew, and M. Buchanan, "Optimized GaAs/AlGaAs lightemitting diodes and high efficiency wafer-fused optical up-conversion devices," J. Appl. Phys, vol. 96, no. 9, pp. 5243-5248, Nov. 2004, doi: 10.1063/1.1785867.

[6] I. Radevici, J. Tiira, T. Sadi, S. Ranta, A. Tukiainen, M. Guina, and J. Oksanen, "Thermophotonic cooling in GaAs based light emitters," Appl. Phys. Lett., vol. 114, no. 5, p. 051101, Feb. 2019, doi: 10.1063/1.5064786.

[7] T. Sadi, I. Radevici, P. Kivisaari, and J. Oksanen, "Electroluminescent Cooling in III-V Intracavity Diodes: Practical Requirements," IEEE Trans. Electron Devices, vol. 66, pp. 963-968, Feb. 2019, doi: 10.1109/TED.2018.2885267.

[8] M. R. Krames, O. B. Shchekin, R. Mueller-Mach, G. O. Mueller, L. Zhou, G. Harbers, and M. G. Craford, "Status and future of highpower light-emitting diodes for solid-state lighting," J. Disp. Technol., vol. 3, no. 2, pp. 160-175, Jun. 2007, doi: 10.1109/JDT.2007.895339.

[9] J. J. Wierer, D. A. Steigerwald, M. R. Krames, J. J. Oshea, M. J. Ludowise, G. Christenson, Y.-C. Shen, C. Lowery, P. S. Martin, S. Subramanya, W. Gtz, N. F. Gardner, R. S. Kern, and S. A. Stockman, "High-power AlGaInN flip-chip light-emitting diodes," Appl. Phys. Lett., vol. 78, no. 22, pp. 3379-3381, Apr. 2001, doi: 10.1063/1.1374499.

[10] T. P. Xiao, K. Chen, P. Santhanam, S. Fan, and E. Yablonovitch, "Electroluminescent refrigeration by ultra-efficient GaAs light-emitting diodes," J. Appl. Phys., vol. 123, no. 17, p. 173104, Mar. 2018, doi: 10.1063/1.5019764

[11] P. Kivisaari, J. Oksanen, and J. Tulkki, "Current injection to freestanding III-N nanowires by bipolar diffusion," Appl. Phys. Lett., vol. 103 , no. 3 , p. 031103 , Jun. 2013 , doi: $10.1063 / 1.4813754$.
[12] L. Riuttanen, P. Kivisaari, H. Nykänen, O. Svensk, S. Suihkonen, J. Oksanen, J. Tulkki, and M. Sopanen, "Diffusion injected multiquantum well light-emitting diode structure," Appl. Phys. Lett., vol. 104, no. 8, p. 081102, Feb. 2014, doi: 10.1063/1.4866343.

[13] L. Riuttanen, P. Kivisaari, O. Svensk, J. Oksanen, and S. Suihkonen, "Diffusion injection in a buried multiquantum well light-emitting diode structure," IEEE Trans. Electron Devices, vol. 62, no. 3, pp. 902-908, Jan. 2015, doi: 10.1109/TED.2015.2391117.

[14] L. Riuttanen, P. Kivisaari, O. Svensk, J. Oksanen, and S. Suihkonen, "Electrical injection to contactless near-surface InGaN quantum well," Appl. Phys. Lett., vol. 107, no. 5, p. 051106, Jul. 2015, doi: 10.1063/1.4928248.

[15] P. Kivisaari, I. Kim, S. Suihkonen, and J. Oksanen, "Elimination of Lateral Resistance and Current Crowding in Large-Area LEDs by Composition Grading and Diffusion-Driven Charge Transport," Adv. Electron. Mat., vol. 3, no. 6, p. 1700103, May 2017, doi: 10.1002/aelm.201700103.

[16] P. Kivisaari, I. Kim, S. Suihkonen, and J. Oksanen, "Elimination of resistive losses in large-area LEDs by new diffusion-driven devices," in Proc. SPIE, vol. 10124, Feb. 2017, p. 101240Z, doi: 10.1117/12.2251108.

[17] M.-L. Lee, Y.-H. Yeh, Z.-Y. Liu, K.-J. Chiang, and J.-K. Sheu, "Planar GaN-based blue light-emitting diodes with surface pn junction formed by selective-area Si-ion implantation," IEEE Trans. Electron Devices, vol. 64, no. 10, pp. 4156-4160, Oct. 2017, doi: 10.1109/TED.2017.2738058

[18] M.-L. Lee, S.-S. Wang, Y.-H. Yeh, P.-H. Liao, and J.-K. Sheu, "Lightemitting diodes with surface gallium nitride $\mathrm{p}-\mathrm{n}$ homojunction structure formed by selective area regrowth," Sci Rep., vol. 9, no. 1, p. 3243, Mar. 2019, doi: 10.1038/s41598-019-40095-7.

[19] C.-W. Cheng, K.-T. Shiu, N. Li, S.-J. Han, L. Shi, and D. K. Sadana, "Epitaxial lift-off process for gallium arsenide substrate reuse and flexible electronics," Nat. Commun., vol. 4, p. 1577, Mar. 2013, doi: 10.1038/ncomms258.

[20] E. Yablonovitch, T. Gmitter, J. Harbison, and R. Bhat, "Extreme selectivity in the lift-off of epitaxial GaAs films," Appl. Phys. Lett., vol. 51, no. 26, pp. 2222-2224, Oct. 1987, doi: 10.1109/JQE.2014.2299752.

[21] R. Windisch, C. Rooman, S. Meinlschmidt, P. Kiesel, D. Zipperer, G. Döhler, B. Dutta, M. Kuijk, G. Borghs, and P. Heremans, "Impact of texture-enhanced transmission on high-efficiency surface-textured lightemitting diodes," Appl. Phys. Lett., vol. 79, no. 15, pp. 2315-2317, Oct. 2001, doi: 10.1063/1.1397758.

[22] D.-M. Yeh, C.-F. Huang, C.-Y. Chen, Y.-C. Lu, and C. Yang, "Localized surface plasmon-induced emission enhancement of a green light-emitting diode," Nanotechnology, vol. 19, no. 34, p. 345201, Aug. 2008, doi: 10.1088/0957-4484/19/34/345201.

[23] M. L. Brongersma, N. J. Halas, and P. Nordlander, "Plasmon-induced hot carrier science and technology," Nat. Nanotechnol., vol. 10, no. 1, p. 25, Jan. 2015, doi: 10.1038/NNANO.2014.311.

[24] A. Olsson, J. Tiira, M. Partanen, T. Hakkarainen, E. Koivusalo, A. Tukiainen, M. Guina, and J. Oksanen, "Optical Energy Transfer and Loss Mechanisms in Coupled Intracavity Light Emitters," IEEE Trans. Electron Devices, vol. 63, no. 9, pp. 3567-3573, Sep. 2016, doi: 10.1109/TED.2016.2590461.

[25] K. Chen, T. P. Xiao, P. Santhanam, E. Yablonovitch, and S. Fan, "Highperformance near-field electroluminescent refrigeration device consisting of a GaAs light emitting diode and a Si photovoltaic cell," J. Appl. Phys., vol. 122 , no. 14 , p. 143104 , Oct. 2017, doi: 10.1063/1.5007712.

[26] P. Santhanam, D. J. Gray, and R. J. Ram, "Thermoelectrically Pumped Light-Emitting Diodes Operating above Unity Efficiency," Phys. Rev. Lett., vol. 108, no. 9, p. 097403, Feb. 2012, doi: 10.1103/PhysRevLett.108.097403.

[27] P. Santhanam, D. Huang, R. J. Ram, M. A. Remennyi, and B. A. Matveev, "Room temperature thermo-electric pumping in mid-infrared light-emitting diodes," Appl. Phys. Lett., vol. 103, no. 18, p. 183513, Oct. 2013, doi: 10.1063/1.4828566.

[28] D. A. Bender, J. G. Cederberg, C. Wang, and M. Sheik-Bahae, "Development of high quantum efficiency GaAs/GaInP double heterostructures for laser cooling," Appl. Phys. Lett., vol. 102, no. 25, p. 252102, Jun. 2013, doi: 10.1063/1.4811759.

[29] K. Lehovec, C. Accardo, and E. Jamgochian, "Light emission produced by current injected into a green silicon-carbide crystal," Phys. Rev., vol. 89 , no. 1 , p. 20 , Jan. 1953, doi: 10.1103/PhysRev.89.20.

[30] J. Tauc, "The share of thermal energy taken from the surroundings in the electro-luminescent energy radiated from a p-n junction," $\mathrm{Ce}$ choslovackij fiziceskij zurnal, vol. 7, no. 3, pp. 275-276, May 1957, doi: 10.1007/BF01688028. 
[31] R. Keyes and T. Quist, "Recombination radiation emitted by gallium arsenide," Proceedings of the Institute of Radio Engineers, vol. 50, no. 8, p. 1822, May 1962, doi: 10.1109/JRPROC.1962.288223.

[32] A. Myllynen, T. Sadi, and J. Oksanen, "Diffusion-driven GaInP/GaAs light-emitting diodes enhanced by modulation doping," Opt. Quant Electron, vol. 51, no. 3, p. 90, Mar. 2019, doi: 10.1007/s11082-0191806-z.

[33] R. Dingle, H. Störmer, A. Gossard, and W. Wiegmann, "Electron mobilities in modulation-doped semiconductor heterojunction superlattices," Appl. Phys. Lett., vol. 33, no. 7, pp. 665-667, Aug. 1978, doi: 10.1063/1.90457.

[34] W. Wang, E. Mendez, and F. Stern, "High mobility hole gas and valence-band offset in modulation-doped p-AlGaAs/GaAs heterojunctions," Appl. Phys. Lett., vol. 45, no. 6, pp. 639-641, May 1984, doi: $10.1063 / 1.95339$.

[35] A. Herzog, D. Keune, and M. Craford, "High-Efficiency Zn-Diffused GaAs Electroluminescent Diodes," J. Appl. Phys., vol. 43, no. 2, pp. 600-608, Aug. 1972, doi: 10.1063/1.1661164.

[36] N. Arnold, R. Schmitt, and K. Heime, "Diffusion in III-V semiconductors from spin-on-film sources," J. Phys. D: Appl. Phys., vol. 17, no. 3, pp. 443-474, Sep. 1984, doi: 0.1088/0022-3727/17/3/006.

[37] J. Donnelly, F. Leonberger, and C. Bozler, "Uniform-carrierconcentration p-type layers in GaAs produced by beryllium ion implantation," Appl. Phys. Lett., vol. 28, no. 12, pp. 706-708, Apr. 1976, doi: $10.1063 / 1.88644$.

[38] T. Sadi, I. Radevici, P. Kivisaari, and J. Oksanen, "Electroluminescent Cooling in III-V Intracavity Diodes: Efficiency Bottlenecks," IEEE Trans. Electron Devices, pp. 2651-2656, Jun. 2019, doi: 10.1109/TED.2019.2910219.

[39] T. Sadi, P. Kivisaari, J. Tiira, I. Radevici, T. Haggren, and J. Oksanen, "Electroluminescent cooling in intracavity light emitters: modeling and experiments," Opt. Quant. Electron, vol. 50, no. 1, p. 18, Jan. 2018, doi: 10.1007/s11082-017-1285-z.

[40] P. Kivisaari, J. Oksanen, J. Tulkki, and T. Sadi, "Monte Carlo simulation of hot carrier transport in III-N LEDs," J. Comput. Electron., vol. 14, no. 2, pp. 382-397, Jun. 2015, doi: 10.1007/s10825-015-0687-z.

[41] P. Kivisaari, T. Sadi, J. Li, P. Rinke, and J. Oksanen, "On the Monte Carlo Description of Hot Carrier Effects and Device Characteristics of III-N LEDs," Adv. Electron. Mat., vol. 3, no. 6, p. 1600494, Jun. 2017, doi: 10.1002/aelm.201600494.

[42] T. Sadi, P. Kivisaari, J. Oksanen, and J. Tulkki, "On the correlation of the Auger generated hot electron emission and efficiency droop in III-N light-emitting diodes," Appl. Phys. Lett., vol. 105, no. 9, p. 091106, Sep. 2014, doi: $10.1063 / 1.4894862$.

[43] Y. Varshni, "Band-to-band radiative recombination in groups IV, VI, and III-V semiconductors (I)," Phys. Status Solidi (B), vol. 19, no. 2, pp. 459-514, 1967, doi: 10.1002/pssb.19670190202.

[44] Y. Varshni, "Band-to-Band Radiative Recombination in Groups IV, VI, and III-V Semiconductors (II)," Phys. Status Solidi (B), vol. 20, no. 1, pp. 9-36, 1967, doi: 10.1002/pssb.19670200102.

[45] U. Strauss, W. W. Rühle, and K. Köhler, "Auger recombination in intrinsic GaAs," Appl. Phys. Lett., vol. 62, pp. 55-57, Jun. 1993, doi: doi.org/10.1063/1.108817.

[46] M. Levinshtein, S. L. Rumyantsev, and M. Shur, Handbook Series on Semiconductor Parameters: Ternary and quaternary III-V compounds. Singapore: World Scientific, Nov. 1999, vol. 2, p. 27, doi: 10.1142/2046vol2. 